\title{
Augmenting Collections of Everyday Objects: A Case Study of Clothes Hangers as an Information Display
}

\author{
Tara Matthews ${ }^{1}$, Hans-Werner Gellersen ${ }^{2}$, Kristof Van Laerhoven ${ }^{2}$, Anind K. Dey ${ }^{3}$ \\ ${ }^{1}$ University of California, Berkeley \\ ${ }^{2}$ Lancaster University \\ ${ }^{3}$ Intel Research Berkeley \\ tmat thewecs.berkeley.edu
}

\begin{abstract}
Though the common conception of human-computer interfaces is one of screens and keyboards, the emergence of ubiquitous computing envisions interfaces that will spread from the desktop into our environments. This gives rise to the development of novel interaction devices and the augmentation of common everyday objects to serve as interfaces between the physical and the virtual. Previous work has provided exemplars of such everyday objects augmented with interactive behaviour. We propose that richer opportunities arise when collections of everyday objects are considered as substrate for interfaces. In an initial case study we have taken clothes hangers as an example and augmented them to collectively function as an information display.
\end{abstract}

\section{Introduction}

The graphical user interface, commonplace on desktops and mobile computers, has been criticized as being too detached from the architectural spaces and physical artefacts around which people's activities evolve. Under headings such as ubiquitous computing, pervasive computing, and ambient intelligence, alternative approaches are investigated that move the interface from the desktop into the environment. One emerging possibility is to augment physical artefacts that already surround us so that they become the interface to an otherwise invisible computing infrastructure. Previous work has produced a range of examples in which everyday artefacts have been augmented with interactive behaviour while retaining their original purpose. These include a picture frame augmented as a context display [1], a table enabled as pointing device [2], and a chest of drawers affording digital lookup of physical content [3].

In this paper, we go beyond the consideration of individual artefacts as interface objects and propose a new type of interface that is based on sets of everyday, physical artefacts that collectively provide interactive behaviour. There are two main reasons to explore this approach. First, many artefacts naturally exist in collections (e.g., CDs, cutlery) and in arrangements (e.g., chairs around a table, pictures on a mantel), a fact that can be taken advantage of for composing interfaces. Second, collections afford additional interactions such as adding, removing, and arranging component artefacts.

Interfaces composed of individual physical entities are of course not a new consideration - interfaces spanning multiple devices have been studied widely in 
traditional user interface research, and there is also a range of examples of tangible interfaces that use the arrangement of physical components as an interaction mechanism (e.g. Triangles [4] and the Urp interface [5]). However, the distinct novelty in considering collections of everyday artefacts as interfaces is that the component artefacts are individually meaningful entities to start with. This gives rise to a host of questions, such as when is an artefact part of an interface and when not, and how can an interface be made robust against any changes in composition that may result from everyday use of component artefacts.

To explore this new interface concept we have focussed on a concrete case study as a starting point. Our approach was to go through the complete process of designing an example interface, of implementing the design as working prototype, and of engaging users with the built prototype to collect formative feedback in addition to our design experience. The example that we have investigated is a Hanger Display. We learned some lessons designing and formatively evaluating the Hanger Display: that existing structures can be useful in defining the interface scope of collections, and that interfaces composed of everyday artefacts must be resilient to change.

\section{Design and Implementation of the Hanger Display}

The Hanger Display is a generic system for portraying information at the periphery of attention. As shown in Figure 1, the Hanger Display consists of a row of hangers, each augmented with a display element (e.g. a light emitting diode, LED), placed on a rod (e.g. in a wardrobe). Collectively the hangers function as a simple display defined by the array of display elements arranged along the rod. The hangers have individual meaning in everyday activity, in the sense that individual hangers are used independently of the state of other hangers (e.g. to hang up a coat, or to move it from one wardrobe to another). But as display elements, they are only meaningful as part of the collection of hangers placed together on the rod.

From an interaction design perspective we think of the Hanger Display as being useful for portraying information that may be relevant to the activities involving hangers (such as hanging and selecting clothes, morning rituals, and getting ready for the day). For example, the hanger display could portray a sense of the day's expected high temperature: given a $0-100^{\circ} \mathrm{F}$ range, if 7 of 10 hangers were lit this would represent $70^{\circ} \mathrm{F}$. However, the Hanger Display does not prescribe how information is mapped onto the variable collection of display elements; it is entirely open-ended for different display purposes. For instance, the hangers may convey any relative quantities (as in the temperature example), progress over time (e.g. in a reminder

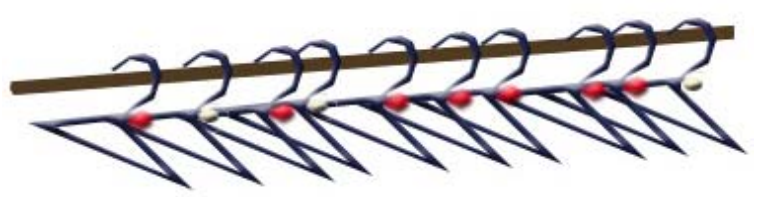

Figure 1: Design Sketch of the Hanger Display. Each of the hangers is augmented with a display element, and all hangers arranged on the rod together function as a coherent display. 
display that counts down days before an important event), or trend information (e.g. analogous to how a barometer displays trends in weather conditions).

We have implemented a working prototype of the Hanger Display based on the following set of components:

- Ordinary hangers augmented with very low-cost embedded hardware (an LED, and a switch to control the LED).

- Hanger rods augmented with a physical network medium to provide connectivity to the hangers.

- Software to manage the display system, i.e. determining which hangers are present on a rod and controlling communication in the system.

- Software mapping an input to the LED output of the Hanger Display, i.e. determining which hangers to turn on and sending commands.

Two aspects are particularly noteworthy in this implementation. First, rods have a distinct role in the design as defining the scope of a display (i.e. determining which artefacts are part of the display and which are not). This is directly reflected in the implementation by foreseeing rods as a connection medium for hangers, in which physical arrangement is overlaid with digital connectivity. Second, the implementation allows the display to be dynamic. Hangers can be removed or inserted without causing the display to break. Instead, the displayed information is re-mapped when the physical composition of the display changes.

Figure 2 shows a photo of the implemented prototype and an illustration of its hardware components. The rod has two strips of aluminium along its length that act as two wires, enabling its use as a network medium. One strip serves as ground and the other provides data and power transmission, using the MicroLAN protocol [6] and a similar networking concept as presented by the Pin\&Play project [7]. In order to connect a hanger to this medium, we put two loops of wire around its hook so that separate contacts are made to the two aluminium strips on the rod. The balance of a hanger, with or without clothing, generally causes it to naturally fall into the correct place on the rod to make these contacts. The hardware on each hanger includes a switch that can be controlled over MicroLAN, an LED whose output is directly connected to the state of the switch, and a resistor to achieve brighter output. The
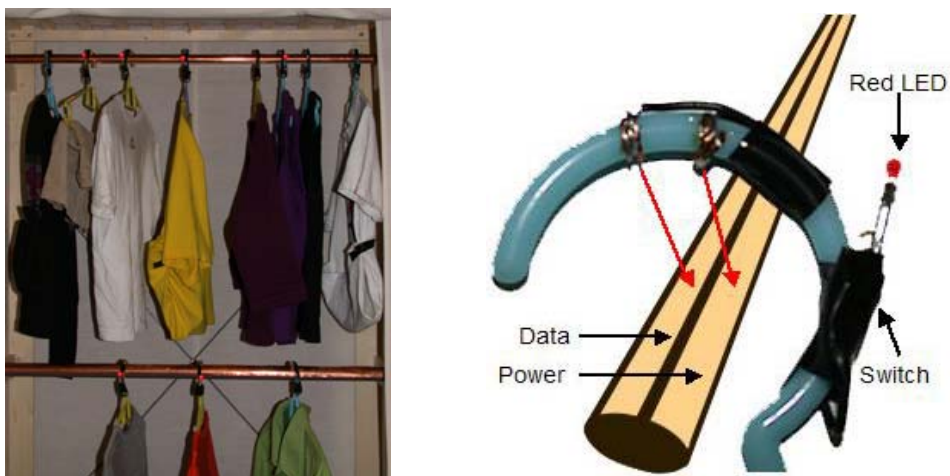

Figure 2: A wardrobe augmented with two built-in Hanger Displays (the top and bottom rods). As detailed on the right, hangers have two wires added that contact the rod through which data and power is provided to the LED and the switch (hidden under black tape). 
Hanger Display software is written in Java. Based on the MicroLAN protocol, it provides core functionality to control the display system, such as detecting which hangers are present on the rod and addressing hangers in order to control the LEDs.

Note that the hardware components are all very small and do not compromise the form-factor of the hangers in any significant way. In addition, despite the fact that only very simple and inexpensive hardware is used for each hanger, the collection of hangers is quite expressive in the way it conveys information.

\section{Formative User Study and Discussion}

In order to obtain formative user feedback we implemented two basic application scenarios in which weather-related information was displayed on the Hanger Display. The applications mapped temperature forecast and the probability of rain to the two rods of our prototype, based on local weather data parsed from the weather.com web site. The intent of this application setup was not to evaluate how effective weather information might be conveyed by such a display, but to engage users in a more general discussion on the use of multiple everyday artefacts as an interface.

To this end, we conducted a formative, in-lab user study with the two applications. We set up a wardrobe as in Figure 2: the top rod displaying the predicted high temperature (the range is $0-30^{\circ} \mathrm{C}$ and the figure shows 4 of 8 hangers are lit, so it will be $15^{\circ} \mathrm{C}$ ), and the bottom rod showing the chance of rain $(2$ of 3 hangers lit, indicating a $66 \%$ chance of rain). Seven researchers from other groups at our department came to the lab for one individual evaluation session each with one of the authors. Participants were given a brief description of the applications and asked to explore and interact with the interface with no specific task. An interview followed, in which the participants were asked for qualitative feedback on the interface. Although feedback was influenced by the participants' previous computing experience, discussions primarily centred on domestic rather than work experience.

The feedback from the formative evaluation showed that there is a realistic use for displays and more generally interfaces composed of multiple everyday, physical artefacts. Every user had ideas for other applications that they would find useful in their own homes. For example, a collection of CDs could be augmented to give you a sense of which CDs you have not listened to recently. When you pick out music, you 
The exciting prospect for examples like these is that they point to new, creative, and useful applications of ubiquitous computing that support activities in situ. Several users pointed out the benefit of an everyday object like hangers displaying additional information: "I like having information in the place where it is useful, so it is nice to get weather information when you are picking out clothes." With further exploration, other collections of everyday objects might suggest new ways to provide the benefits of technology without sacrificing the ease and naturalness of everyday activities.

The formative feedback also helped confirm some design considerations for interfaces composed of multiple everyday, physical artefacts. The first consideration is that existing structures can be useful in defining interface scope. For the Hanger Display, the natural affordances of the rod made it easy for users to understand the interface and to determine which hangers were part of it.

The second consideration is that interfaces composed of everyday artefacts must be resilient to change. Aside from some problems with the prototype implementation (e.g., hangers not always making a connection with the rod), the users asserted that the embedded technology did not interfere with natural interaction. The Hangers show we can design to deal with physical re-composition, but future work is needed to investigate how this can be supported in a general way.

\section{Conclusion}

In this paper, we have presented a case study of an interface composed of a collection of everyday artefacts, which attempts to provide information in the context of everyday activities and environments. The design of and formative feedback on the Hanger Display shows that this approach is interesting for two main reasons: many artefacts naturally exist in collections; and collections afford additional interactions such as adding, removing, and arranging of component artefacts. When designing such interfaces, we learned that existing structures can be useful in defining interface scope, and that interfaces composed of everyday artefacts must be resilient to change.

\section{References}

1. Mynatt, E.D., et al. Digital family portraits: Providing peace of mind for extended family members. Proc. of ACM CHI'01, pp. 333-340.

2. Schmidt, A., et al. Ubiquitous interaction - Using surfaces in everyday environments as pointing devices. Proc. $7^{\text {th }}$ Workshop on User Interfaces for All, 2002, pp. 263-279.

3. Siio, I., et al. Finding Objects in 'Strata Drawer.' Extended Abstracts CHI '03, pp. 982-983.

4. Gorbett, M.G., et al. Triangles: Tangible interface for manipulation and exploration of digital information topography. Proc. of ACM CHI'98, pp. 49-56.

5. Underkoffler, J. and Ishii, H. Urp: A luminous-tangible workbench for urban planning and design. Proc. of ACM CHI'99, pp. 386-393.

6. Dallas Semiconductors. Overview of 1-Wire Techn. \& its Use. App Note 1796, Dec 3, '03.

7. Van Laerhoven, K., et al. Pin\&Play: Networking Objects Through Pins. Proc. of Ubicomp '02, pp. 219-229. 